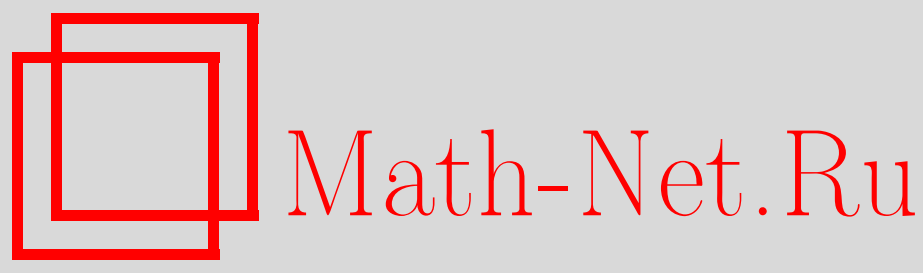

В. В. Рязанов, Получение термодинамических соотношений для гиббсовского ансамбля при помощи метода максимума энтропии, ТМФ, 2018, том 194, номер 3, 452467

DOI: https://doi.org/10.4213/tmf9333

Использование Общероссийского математического портала Math-Net.Ru подразумевает, что вы прочитали и согласны с пользовательским соглашением http://www.mathnet.ru/rus/agreement

Параметры загрузки:

IP: 52.6 .47 .48

26 апреля 2023 г., 16:06:56

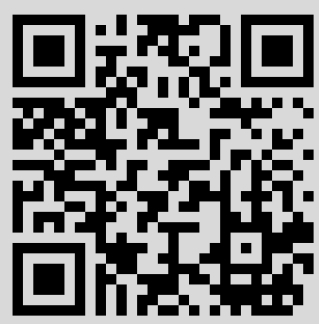




\title{
ПОЛУЧЕНИЕ ТЕРМОДИНАМИЧЕСКИХ СООТНОШЕНИЙ ДЛЯ ГИББСОВСКОГО АНСАМБЛЯ ПРИ ПОМОЩИ МЕТОДА МАКСИМУМА ЭНТРОПИИ
}

\begin{abstract}
В качестве производящего функционала гиббсовского ансамбля используется результат преобразования Лапласа сложной (или обобщенной) меры Пуассона. Для определения вида производящей функции этого распределения используется принцип максимума энтропии. Рассмотрены случаи, когда известно только математическое ожидание и когда известны математическое ожидание и второй момент. В последнем случае уравнение состояния имеет трансцендентную форму. В обоих случаях в отсутствии взаимодействия полученные соотношения приводят к выражениям для идеального газа.
\end{abstract}

Ключевые слова: гиббсовские системы, большой канонический ансамбль, обобщенное распределение Пуассона, принцип максимума энтропии.

DOI: https://doi.org/10.4213/tmf9333

\section{1. ВВЕДЕНИЕ}

Уравнение состояния связывает между собой термодинамические (макроскопические) параметры системы, такие как температура, давление, объем, химический потенциал и др. Соотношения, задаваемые уравнением состояния, справедливы для термодинамического описания равновесных состояний. Уравнения состояния в случае реальных систем могут быть крайне сложными, и их нельзя вывести из термодинамики. Уравнение состояния должно быть взято со стороны (из опыта или из модели, созданной в рамках статистической физики). Последнему вопросу и посвящена настоящая работа.

Уравнение состояния и термодинамические свойства веществ важны с точки зрения самых различных применений. Известно большое число уравнений состояния, в основном эмпирических. Строгий вывод такого рода соотношений из статистической физики в научной литературе не так распространен. Одной из последних работ в этом направлении можно назвать статью [1], но в ней, в отличие от настоящей работы, уравнение состояния записывается в виде разложения, и уравнение Ван-дер-Ваальса, имеющее форму конечного замкнутого выражения, также разлагается в ряд.

${ }^{*}$ Институт ядерных исследований НАН Украины, Киев, Украина.

E-mail: vryazan@yandex.ru 
В настоящей работе, кроме применения распределений Гиббса для классических частиц с парным короткодействующим потенциалом взаимодействия, используются такие общие подходы, как принцип максимума энтропии, метод производящего функционала (ПФ) и обобщенные распределения Пуассона. В работах [2] был предложен формализм восстановления неизвестных законов распределения случайных величин из условий максимума энтропии Шеннона при наличии ограничений. Принцип максимума энтропии применялся к огромному количеству разнообразных систем. Обзоры этой обширной темы даны в работах [3]. Понятие ПФ в статистическую физику было введено Боголюбовым [4]. ПФ чаще использовался в квантовой теории поля, но он применялся и к другим задачам [5], [6]. В работе [6] использовался вариационный принцип. Обзор работ, посвященных ПФ и написанных до 1984 г., можно найти в [7]. Определения ПФ в статьях [7] и в [8], [9] несколько отличаются от определения ПФ в [4], но они могут быть сведены друг к другу. Это же относится и к ПФ в форме, предложенной в работах [10], [11], взятой из общего определения ПФ точечных случайных процессов (см., например [12], [13]). ПФ из работ [10], [11] применялся в статьях [14]-[16] для вывода уравнений состояния. Связь обобщенного распределения Пуассона и задач статистической физики рассмотрена в ряде работ начиная с [17]. Идеи этой работы используются в [18], [19], они связаны и с кластерными разложениями, и с преобразованиями синус-гордона [20]. В работе [19] представлен ряд новых примеров моделей движения и начальных случайных точечных полей, для которых имеет место сходимость к пуассоновским случайным точечным полям. Можно отметить также работы [21], [22], где определена вероятностная мера на конфигурациях в большом каноническом ансамбле, хотя автор работы [22] и не называет ее мерой Пуассона. Подробный обзор связи между мерой Пуассона и вероятностными мерами Гиббса был проведен в [23]. В работе [24] ПФ был выражен через меры Лебега-Пуассона; также ряд результатов о мерах Лебега-Пуассона содержится в [25], [26].

Работа организована следующим образом. В разделе 2 мы применяем метод максимума энтропии к ПФ обобщенного распределения Пуассона в случае, когда заданы только условие нормировки распределения и математическое ожидание. В разделе 3 сформулирован основной результат работы: для случая, когда известны нормировка, математическое ожидание и второй момент, выведены следствия из применения метода максимума энтропии к ПФ обобщенного распределения Пуассона.

\section{2. УРАВНЕНИЕ СОСТОЯНИЯ ПРИ ИЗВЕСТНОМ ПЕРВОМ МОМЕНТЕ}

Мы рассматриваем систему, подчиняющуюся классическим законам Ньютона. Пусть $\mathbb{R}^{d}-d$-мерное евклидово пространство. Рассматривается система из $n$ частиц в области $\Lambda$ физического пространства $\mathbb{R}^{d}$ (далее мы ограничимся случаем $d=3)$. Фазовое пространство такой системы представляет собой множество размерности $2 n d$. Чтобы указать точку этого фазового пространства, надо задать $n$ точек $q_{1}, \ldots, q_{n}$ области $\Lambda$ и $n$ импульсов $p_{1}, \ldots, p_{n}$, принадлежащих $\mathbb{R}^{d}$ [27]. Если обозначить $\left(q_{1}, \ldots, q_{n}\right)$ через $\vec{q},\left(p_{1}, \ldots, p_{n}\right)$ - через $\vec{p},\left(q_{i}, p_{i}\right) \in \Lambda \times \mathbb{R}^{d}$ - через $x_{i}$, a $\left(q_{1}, p_{1}, \ldots, q_{n}, p_{n}\right)$ - через $\vec{x}$, то энергия, отвечающая фазовой точке $\vec{x}$, равна

$$
H(\vec{x})=T(\vec{p})+U(\vec{q}), \quad T(\vec{p})=\frac{1}{2 m} \sum_{i} p_{i}^{2}, \quad U(\vec{q})=\sum_{i<j} V\left(q_{i}-q_{j}\right),
$$


где $V(x-y)=\Phi_{2}(|x-y|), x, y \in \Lambda, \Phi_{2}$ - парный потенциал взаимодействия частиц, $m$ - масса частицы. Предположим, что потенциальная энергия $U\left(q_{1}, \ldots, q_{n}\right)=U_{n}$ системы из $n$ частиц с гамильтонианом (1) термодинамически устойчива, т. е. существует такая константа $B$, что $U\left(q_{1}, \ldots, q_{n}\right) \geqslant-n B$ для всех $n$ и всех $q_{1}, \ldots, q_{n} \in \mathbb{R}^{d}$. В работах [27] это требование дополняется условием сверхустойчивости. Разобьем координатное пространство на единичные кубы с центрами в точках $\mathbb{Z}^{d}$. Для $\alpha \in \mathbb{Z}^{d}$ обозначим такой куб с центром в $\alpha$ через $\Delta(\alpha)$, а для $\vec{x} \in \mathfrak{X}$ обозначим через $n_{\alpha}(\vec{x})$ число частиц в $\Delta(\alpha)$. Здесь множество $\mathfrak{X}(\Lambda)$ есть объединение по всем $n$ фазовых пространств $n$-частичных систем, заключенных в $\Lambda$,

$$
\mathfrak{X}(\Lambda)=\bigcup_{n=0}^{\infty}\left(\Lambda \times \mathbb{R}^{d}\right)_{\text {symm }}^{n} .
$$

Потенциал $\Phi_{2}$ сверхустойчив, если существуют константы $A, B>0$ такие, что

$$
U(\vec{q}) \geqslant-B n_{\alpha}(\vec{q})+A \sum_{\alpha} n_{\alpha}^{2}(\vec{q})
$$

для всех $n$ и всех $q_{1}, \ldots, q_{n}[28]$. На потенциал взаимодействия налагается условие интегрируемости на бесконечности

$$
\int_{|x| \geqslant R} \Phi_{2}(|x|) d x<+\infty
$$

где $R>0$ произвольно, а также условие регулярности

$$
C(\beta)=\int_{\mathbb{R}^{d}}\left|e^{-\beta \Phi_{2}(|x|)}-1\right| d x<\infty .
$$

В работах [23], [25], [29], [30] были также сформулированы условие усиленной сверхустойчивости потенциальной энергии и условия, налагаемые на потенциал взаимодействия.

Предположим, что задано вещественное число $\mu$, называемое химическим потенциалом, и параметр $\beta,-\infty<\beta<\infty$, обратно пропорциональный температуре системы. В рассмотрение легко включить внешнее поле, которое будет добавкой к химическому потенциалу. Следуя [27], выберем значения обратной температуры $\beta$ и химического потенциала $\mu$ и для каждого ограниченного множества $\Lambda$ зададим вероятностную меру ${ }_{\Lambda} \mu$ (которая называется большой канонической вероятностной мерой, или большим каноническим ансамблем) на пространстве $\mathfrak{X}(\Lambda)$ формулой

$$
d_{\Lambda} \mu=\frac{e^{-\beta H\left(\vec{x}_{\Lambda}\right)+\beta \mu n\left(\vec{x}_{\Lambda}\right)} d \vec{x}_{\Lambda}}{\Xi(\Lambda, \beta, \mu)}, \quad \vec{x}_{\Lambda} \in \Lambda,
$$

где энергия $H(\vec{x})$ определена в $(1), k$ - постоянная Больцмана, $T$ - абсолютная температура, $\beta=1 / k T$ и

$$
\begin{aligned}
\Xi(\Lambda, \beta, \mu) & =\int e^{-\beta H\left(\vec{x}_{\Lambda}\right)+\beta \mu n\left(\vec{x}_{\Lambda}\right)} d \vec{x}_{\Lambda}= \\
& =\sum_{n=0}^{\infty} \frac{1}{n !} e^{\beta \mu n}\left(\frac{2 \pi m}{\beta}\right)^{n d} \int_{\Lambda^{n}} e^{-\beta U\left(q_{1}, \ldots, q_{n}\right)} d q_{1} \ldots d q_{n} .
\end{aligned}
$$


Мы рассматриваем конечные системы, но можно перейти к пределу бесконечных систем. Более детальное описание мер Гиббса и предельного перехода к бесконечным системам можно найти в [31], [32].

ПФ случайной меры $\nu$ в теории случайных процессов определяется как [12]

$$
F(s(\cdot))=E\left\{\exp \int_{V} \ln s(x) \nu(d x)\right\}
$$

где $E\{\cdot\}$ обозначает усреднение по всем возможным реализациям случайной функции $d \nu / d x, s(\cdot)$ - аргумент ПФ. ПФ существует, если $|s(x)| \leqslant 1$ и $\int \ln s(x) \nu(d x)$ существует.

Пусть $(\Omega, \mathfrak{X}, \mathbb{P})$ - некоторое вероятностное пространство и каждому $\omega \in \Omega$ соответствует счетная последовательность действительных чисел $\left\{x_{i}\right\}$ без предельных точек. Эта последовательность называется точечным процессом. Для точечных процессов определение ПФ (4) сформулировано в книге [13]. В работе [7] отмечается, что практически все результаты, полученные для ПФ конфигурационных мер непрерывных моделей, автоматически переносятся на конфигурационные меры дискретных моделей.

Если задавать частицы как точки, лишенные объема и внутренней структуры, то точечная мера, описывающая их число, имеет вид

$$
\nu(d x)=\sum_{i=1}^{N} \delta\left(x_{i}-x\right) d x
$$

где $N$ - случайная величина. В работе [8] показано, что для конечных систем метод ПФ эквивалентен методу распределения Гиббса в большом каноническом ансамбле. Подставляя (5) в (4), получим

$$
F(s)=E\left\{\prod_{i=1}^{N} s\left(x_{i}\right)\right\}=\sum_{n=0}^{\infty} \int_{V} \ldots \int_{V} D_{n}\left(x_{1}, \ldots, x_{n}\right) \prod_{i=1}^{n} s\left(x_{i}\right) d x_{i},
$$

где $D_{n}\left(x_{1}, \ldots, x_{n}\right)=d_{\Lambda} \mu / d \vec{x}_{\Lambda}-$ вероятностные меры для большого канонического ансамбля, заданные в формулах $(2),(3)$. Мы рассматриваем системы с парным взаимодействием частиц одного типа. Потенциал взаимодействия $\Phi_{2}(x), x \in \mathbb{R}^{n}$, есть измеримая четная функция, которая может принимать действительные значения и значение $+\infty$.

Для равновесных систем все наблюдаемые величины являются функциями координат. Поэтому, подставив соотношения (2), (3) для большой канонической вероятностной меры в (6), можно выполнить интегрирование по импульсным переменным $p_{i} \in \mathbb{R}^{d}, i=1, \ldots, n$, как в (3), и переписать выражение (6) в виде

$$
\begin{aligned}
F(s) & =E\left\{\prod_{i=1}^{N} s\left(x_{i}\right)\right\}=\sum_{n=0}^{\infty} \int_{V} \cdots \int_{V} e^{\left(n \mu-H_{n}\right) / k T} \Xi^{-1} \prod_{i=1}^{n} s\left(x_{i}\right) d x_{i}= \\
& =\sum_{n=0}^{\infty} \int_{V} \cdots \int_{V} z^{n} e^{-\beta U\left(q_{1}, \ldots, q_{n}\right)} \Xi^{-1} \prod_{i=1}^{n} s\left(q_{i}\right) d q_{i},
\end{aligned}
$$

где

$$
H\left(\vec{x}_{\Lambda}\right)=H_{n}\left(x_{1}, \ldots, x_{n}\right), \quad D_{n}=e^{\left(n \mu-H_{n}\right) / k T} \Xi^{-1} .
$$


При этом

$$
Q_{n}=\frac{1}{h^{3 n} n !} \int_{V} \ldots \int_{V} e^{-H_{n} / k T} d x_{1} \ldots d x_{n}
$$

есть статистическая сумма гиббсовского канонического ансамбля из $n$ частиц, $H_{n}-$ энергия системы из $n$ частиц (1) и большая статистическая сумма (3) записывается каK

$$
\Xi=\sum_{n=0}^{\infty} \frac{z^{n}}{n !} Q_{n}
$$

где $z_{i}=(2 \pi m k T)^{3 / 2} h^{-3} e^{\mu_{i} / k T}-$ значение активности в $i$-й точке (здесь $h$ - постоянная Планка и предполагается аддитивность взаимодействия). Для вероятностных мер Гиббса и большого канонического ансамбля $e^{-\beta \Phi_{2}(x)}-1 \equiv f(x)-$ функции Майера [7], [33], [34]. Из (6), (7) получаем

$$
\begin{aligned}
& \left.\frac{\delta^{k} F(s)}{\delta s_{1} \ldots \delta s_{k}}\right|_{s=0}=k ! D_{k}, \\
& \left.\frac{\delta^{k} F(s)}{\delta s_{1} \ldots \delta s_{k}}\right|_{s=1}=\rho^{(k)}, \quad k=1,2, \ldots,
\end{aligned}
$$

где $s_{i}=s\left(x_{i}\right)$, а $\rho^{(k)}$ - факториальные моменты $k$-го порядка случайной меры $\nu$. При усреднении по гиббсовскому ансамблю $\rho^{(k)}$ совпадают с функциями распределения $k$-го порядка [33].

Для ПФ (6)-(8) точечной случайной меры числа частиц (5) с усреднением по большому каноническому ансамблю (2), (3) справедливы соотношения [4]

$$
\begin{gathered}
\frac{\delta^{k} F(s)}{\delta s_{1} \ldots \delta s_{k}}=z_{1} \ldots z_{k} e^{-U_{k} / k T} F\left[s(x) \prod_{i=1}^{k} y_{x_{i} x}\right], \quad \frac{\delta F(s)}{\delta s\left(x_{1}\right)}=z\left(x_{1}\right) F\left[s(x) y_{x_{1} x}\right], \\
\rho_{1}^{(1)}=z_{1} F\left(y_{x_{1} x}\right),
\end{gathered}
$$

где $y_{x_{i} x_{j}}=e^{-\Phi_{2}\left(x_{i}-x_{j}\right) / k T}=f\left(x_{i}-x_{j}\right)+1$. Они получаются непосредственно, если подставить меру Гиббса (2) большого канонического ансамбля в (6) и применить к полученному выражению функциональное дифференцирование с учетом определения активности и аддитивности парного взаимодействия.

Следуя [35], определим конфигурационное пространство и меру Лебега-Пуассона. Обозначим через $\mathscr{B}\left(\mathbb{R}^{d}\right)$ борелевскую $\sigma$-алгебру открытых множеств в $\mathbb{R}^{d}$, а через $\mathscr{B}_{\mathrm{c}}\left(\mathbb{R}^{d}\right)$ - класс всех ограниченных множеств из $\mathscr{B}\left(\mathbb{R}^{d}\right)$. Конфигурационное пространство $\Gamma=\Gamma_{\mathbb{R}^{d}}$ состоит из всех локально конечных подмножеств пространства $\mathbb{R}^{d}$,

$$
\Gamma=\Gamma_{\mathbb{R}^{d}}=\left\{\gamma \subset \mathbb{R}^{d}:|\gamma \cap \Lambda|<\infty \text { для всех } \Lambda \in \mathscr{B}_{\mathrm{c}}\left(\mathbb{R}^{d}\right)\right\},
$$

где для множества $A \in \mathscr{B}_{\mathrm{c}}\left(\mathbb{R}^{d}\right)$ мы обозначили через $|\mathrm{A}|$ число его точек. Обозначим множество всех конечных конфигураций пространства $Г$ через $\Gamma_{0}$. Пространство конечных конфигураций, которые находятся в некотором ограниченном множестве $\Lambda \in \mathscr{B}_{\mathrm{c}}\left(\mathbb{R}^{d}\right)$, обозначим как $\Gamma_{\Lambda}$. Конфигурационное пространство с фиксированным числом элементов будем обозначать как $\Gamma^{(n)}$. 
Пусть $\sigma$ - мера Лебега в $\mathbb{R}^{d}$. Состояние идеального газа в равновесной статистической механике описывается мерой Пуассона $\pi_{z \sigma}$ на конфигурационном пространстве Г, где $z>0$ - это активность. Для любого $z>0$ определим меру Лебега-Пуассона $\lambda_{z \sigma}$ на $\mathscr{B}\left(\Gamma_{0}\right)$ формулой

$$
\lambda_{z \sigma}=\sum_{n=0}^{\infty} \frac{z^{n}}{n !} \sigma^{(n)} .
$$

Интеграл по мере $\lambda_{z \sigma}$ задается как

$$
\int_{\Gamma_{\Lambda}} F(\gamma) \lambda_{z \sigma}(d \gamma)=\sum_{n=0}^{\infty} \frac{z^{n}}{n !} \int_{\Lambda} \ldots \int_{\Lambda} F_{n}\left(x_{1}, \ldots, x_{n}\right) d x_{1} \ldots d x_{n}
$$

для всех измеримых функций $F=\left\{F_{n}\right\}_{n \geqslant 0}, F_{n} \in L^{\infty}\left(\Lambda^{n}\right)\left(\right.$ или $\left.F_{n} \in L^{1}\left(\mathbb{R}^{d n}\right)\right)$. При помощи мер $\lambda_{z \sigma}$ построим семейство вероятностных мер

$$
\pi_{z \sigma}^{\Lambda}=e^{-z \sigma(\Lambda)} \lambda_{z \sigma}^{\Lambda}, \quad \Lambda \in \mathscr{B}_{\mathrm{c}}\left(\mathbb{R}^{d}\right) .
$$

Меры этого семейства попарно согласованы. Существует вероятностная мера Пуассона $\pi_{z \sigma}$ на конфигурационном пространстве $\Gamma$, которая определяется следующим образом:

$$
\pi_{z \sigma}^{\Lambda}=\pi_{z \sigma} \circ\left(p_{\Lambda}\right)^{-1}, \quad p_{\Lambda}: \Gamma \mapsto \Gamma_{\Lambda} \quad \text { для каждого } \Lambda \in \mathscr{B}_{\mathrm{c}}\left(\mathbb{R}^{d}\right)
$$

Вероятностную меру $\pi_{\sigma}$ называют мерой Пуассона с интенсивностью $\sigma$. Пространство мер $\left(\Gamma, \mathscr{B}_{\mathrm{c}}\left(\mathbb{R}^{d}\right), \pi_{\sigma}\right)$ называется пуассоновским пространством.

Для определения преобразования Лапласа меры Пуассона $\pi_{\sigma}$ рассмотрим функцию $f \in C_{0}(X)$. Носитель функции $f$ содержится в некотором объеме $\Lambda \in \mathscr{B}_{\mathrm{c}}\left(\mathbb{R}^{d}\right)$, поэтому получаем

$$
\langle\gamma, f\rangle=\left\langle p_{\Lambda}(\gamma), f\right\rangle, \quad \gamma \in \Gamma
$$

Преобразование Лапласа меры $\pi_{\sigma}$ задается интегралом

$$
\begin{aligned}
l_{\pi_{\sigma}}(f) & =\int_{\Gamma} e^{\langle\gamma, f\rangle} \pi_{\sigma}(d \gamma)=\int_{\Gamma} e^{\left\langle p_{\Lambda}(\gamma), f\right\rangle} \pi_{\sigma}(d \gamma)= \\
& =\int_{\Gamma_{\Lambda}} e^{\langle\gamma, f\rangle} \pi_{\sigma}^{\Lambda}(d \gamma)=e^{-\sigma(\Lambda)} \int_{\Gamma_{\Lambda}} e^{\langle\gamma, f\rangle} \lambda_{\sigma}^{\Lambda}(d \gamma)= \\
& =e^{-\sigma(\Lambda)} \exp \left\{\int_{\Lambda} e^{f(x)} \sigma(d x)\right\}=\exp \left\{\int_{\mathbb{R}^{d}}\left(e^{f(x)}-1\right) \sigma(d x)\right\} .
\end{aligned}
$$

При помощи меры Лебега-Пуассона (13) зададим меру Гиббса (2), (3) большого канонического ансамбля в конечном объеме:

$$
\mu_{\Lambda}(d \gamma)=\frac{1}{Z_{\Lambda}} e^{-\beta U(\gamma)} \lambda_{z \sigma}(d \gamma), \quad Z_{\Lambda}=\int_{\Gamma_{\Lambda}} e^{-\beta U(\gamma)} \lambda_{z \sigma}(d \gamma) .
$$

В работе [20] рассматривалась $m$-частичная функция распределения (10) в конечном объеме $\Lambda$ для большого канонического ансамбля:

$$
\rho^{\Lambda}(x)_{m}=\frac{1}{Z_{\Lambda}} \sum_{n \geqslant m} \frac{z^{n}}{(n-m) !} \int_{\Lambda^{n-m}} e^{-\beta U(x)_{n}}(d x)_{n \backslash m},
$$


где $(x)_{m}=\left(x_{1}, \ldots, x_{m}\right),(d x)_{n \backslash m}=d x_{m+1} \ldots d x_{n}$,

$$
U(x)_{n}=\sum_{1 \leqslant i<j \leqslant n} V\left(x_{i}-x_{j}\right)
$$

и $V(x-y)=\Phi_{2}(|x-y|)$ - парный потенциал с условием регулярности $V(0)=v_{0}<\infty$. В работе [20] было выполнено преобразование синус-гордона

$$
\begin{gathered}
e^{-\beta U(x)_{n}}=e^{1 / 2 \beta n v_{0}} \int \exp \left\{i \beta^{1 / 2} \sum_{1 \leqslant j \leqslant n} \Phi\left(x_{j}\right)\right\} d \mu(\Phi), \\
Z_{\Lambda}=\int \exp \left\{\tilde{z} \int_{\Lambda} e^{i \beta^{1 / 2} \Phi(x)} d x\right\} d \mu(\Phi), \quad \tilde{z}=z e^{1 / 2 \beta v_{0}} .
\end{gathered}
$$

Благодаря условию $V(0)=v_{0}<\infty$ гауссовская мера $d \mu(\Phi)$ определена на измеримом пространстве непрерывных функций $\Phi(x), x \in \Lambda$. Положим $Z(\Lambda)=Z_{\Lambda} e^{-\tilde{z}|\Lambda|}$, тогда $m$-частичная функция распределения переписывается в виде

$$
\rho^{\Lambda}(x)_{m}=\tilde{z}^{m} \frac{1}{Z(\Lambda)} \int \exp \left\{i \beta^{1 / 2} \sum_{j} \Phi\left(x_{j}\right)\right\} \exp \left\{\tilde{z} \int_{\Lambda}\left(e^{i \beta^{1 / 2} \Phi(x)}-1\right) d x\right\} d \mu(\Phi),
$$

В правой части этого выражения фигурирует характеристический функционал некоторого обобщенного процесса Пуассона. Определим его.

Пусть неотрицательная конечная мера $\tau$ на $\mathbb{R}_{+}=(0, \infty)$ имеет конечные моменты и удовлетворяет свойству аналитичности: найдется $C>0$ такое, что для всех достаточно больших $n$

$$
\int_{0}^{\infty} s^{n} d \tau(s)<C^{n} n !
$$

Пусть $X$ - связное, ориентированное (некомпактное) риманово $C^{\infty}$-многообразие. Обозначим через $D=C_{0}^{\infty}(X)$ пространство бесконечно дифференцируемых функций с компактным носителем. Мера $\pi_{\sigma}^{\tau}$ на $D^{\prime}\left(D^{\prime}-\right.$ дуальное по отношению к $D$ пространство) называется сложной (или обобщенной) мерой Пуассона, если ее преобразование Лапласа для $\varphi \in D$ имеет вид [36]

$$
l_{\pi_{\sigma}^{\tau}}(\varphi)=\int_{D^{\prime}} e^{\langle\omega, \varphi\rangle} d \pi_{\sigma}^{\tau}(\omega)=\exp \left\{\int_{X} \int_{0}^{\infty}\left(e^{\alpha \varphi(x)}-1\right) d \tau(\alpha) d \sigma(x)\right\} .
$$

Ниже мы полагаем, что $\alpha$ - целочисленная неотрицательная случайная величина. В работе [37] это соотношение записано в виде

$$
L_{\tau}(\varphi)=\exp \left\{\int_{X} \ln \chi_{\tau}(\varphi(x)) d \sigma(x)\right\}=\exp \left\{\int_{\mathbb{R}} \int_{X}\left(e^{i \alpha \varphi(x)}-1\right) d \sigma(x) d \tau(\alpha)\right\},
$$

где $\chi_{\tau}-$ положительно определенная функция на $\mathbb{R}$ вида

$$
\chi_{\tau}(t)=\exp \left\{\int_{-\infty}^{\infty}\left(e^{i \alpha t}-1\right) d \tau(\alpha)\right\}
$$

в формулах (17), (18) $\tau$ - неотрицательная конечная (не обязательно нормированная) мера на $\mathbb{R}, \sigma$ - фиксированная гладкая положительная мера на $X$. 
Значения $m$-частичных функций распределения (15) определяют систему вероятностных мер Гиббса (2), (3), (14), корреляционные функции связаны с плотностями распределения и конфигурационными мерами [7], [22]. Значения ПФ тоже, как показано в [7], [24], определяют вероятностные меры и характеризуют состояние равновесной гиббсовской системы. В [35] меры Гиббса (меры Рюэля) были представлены в виде сложных мер Пуассона.

В работах [24], [26] было показано, что ПФ является также ПФ корреляционных функций (15), которые непрерывны относительно вероятностных мер Пуассона [20]. Это также вытекает из (10). Вероятностная мера Гиббса и статистическая сумма большого канонического ансамбля были представлены как преобразование меры Лебега-Пуассона также в работе [25]. Мы запишем ПФ гиббсовской системы (7) как ПФ обобщенного распределения Пуассона.

В выражениях (16), (17) предположим, что $\tau(\alpha)$ - вероятностная мера, фигурирующая в выражении (18). Пусть она имеет дискретный решетчатый характер и нормирована, $\int_{0}^{\infty} d \tau(\alpha)=1$. Проведем в выражении (16) интегрирование по $\alpha$, совершим замену аргумента $\varphi(x) \rightarrow \ln s(x)$ при $d \tau(\alpha)=\tau_{k} \delta(\alpha-k) d \alpha, \tau_{k}=\left.\tau(\alpha)\right|_{\alpha=k}$, $k=1, \ldots, n$, перейдем от характеристической функции к производящей функции $p(s)=\sum_{k=1}^{n} s^{k} \tau_{k}$. Далее переобозначим $\tau_{k}=p_{k}$ и совершим замены $x \rightarrow r, X \rightarrow V$, где $r$ - пространственная переменная в объеме $V$. Наконец, предположим, что мера $\sigma$ имеет плотность $a(r)$ относительно меры Лебега на пространстве $X$, при этом $\left.d \sigma(x)\right|_{x=r}=a(r) d r$, и заменим левые части равенств (16) и (17) на $F(s)$. В результате выражения (16) и (17) принимают вид

$$
\ln F(s)=\int_{V}[p(s(r))-1] a(r) d r .
$$

Сопоставим друг другу ПФ (7) и (19). Для обобщенного или для сложного распределения Пуассона (сложный процесс Пуассона совпадает с обобщенным, если $\alpha-$ целочисленная случайная величина [38]) вероятности $p_{k}=\left.\tau(\alpha)\right|_{\alpha=k}$ из выражения $p(s)=\sum_{k=1}^{n} s^{k} p_{k}$ характеризуют поведение последовательности независимых одинаково распределенных случайных величин $\xi_{1}, \xi_{2}, \ldots$, при этом случайная величина $\xi=\sum_{k=1}^{\nu} \xi_{k}$, где $\nu$ имеет распределение Пуассона с параметром $a$, описывается сложным распределением Пуассона. Величина $\xi$ сопоставляется со случайным числом частиц в гиббсовской системе. Производящая функция $p(s)$ из $(19)$ описывает число частиц в одном скоплении $\xi_{k}$. В скоплениях $\xi_{k}$ не учитываются вероятности $p_{0}$ (вклад скоплений нулевой величины), размер их также ограничен сверху некоторым числом $n$, это отражено в виде производящей функции $p(s)$.

Общность выражений (16), (17), (19) связана также с тем, что характеристическая функция обобщенного распределения Пуассона представляет собой частный случай канонической формы безгранично делимых распределений при условии неотрицательности случайной меры $\nu$, число частиц (5) неотрицательно. В такой форме в [36] записано выражение (16). Наиболее общий класс распределений с известной в явном виде характеристической функцией - это безгранично делимые распределения. Они также являются самыми общими предельными распределениями, играющими важную роль в физике. Переход от производящей функции к ПФ соответствует обобщению на неоднородные системы. 
Будем искать явный вид функции $p(s)$ в (19), используя принцип максимума энтропии [2]. Для этого запишем функционал Лагранжа, содержащий выражение для энтропии и известные ограничения, и найдем распределение, которое доставляет экстремум функционала. Применим этот принцип к определению вероятностей $p_{k}$, задающих $p(s)$ в (19). Предположим, что заданы условие нормировки распределения и среднее значение $m$ по этому распределению, и будем искать экстремум функционала

$$
L=H+\lambda_{1}\left(\sum_{i=1}^{n} p_{i}-1\right)+\lambda_{2}\left(\sum_{i=1}^{n} x_{i} p_{i}-m\right), \quad H=-\sum_{i=1}^{n} p_{i} \ln p_{i},
$$

где $\lambda_{1}, \lambda_{2}$ - множители Лагранжа. Максимизация этого функционала с помощью дифференцирования его по $p_{i}$ дает

$$
p_{i}=\frac{e^{\lambda_{2} x_{i}}}{\sum_{k=1}^{n} e^{\lambda_{2} x_{k}}} .
$$

Это распределение Гиббса в терминах статистической физики, а в терминологии теории вероятностей - геометрическое распределение в дискретном случае или показательное распределение в непрерывном случае.

Ниже будут использоваться обозначения из [33]. Так как в (7) и (19) проводится интегрирование по объему, в формуле (19) $a=P / k T$. Это следует из соотношений (8), (9) при $s=0$ и $k=0$ и из (19): $\ln F(0)=-\ln \Xi=-P V / k T$, где $P$ давление, $V$ - объем. При $\lambda_{2}<0$ из выражения (20), умножая его на $s^{i}$ и проводя суммирование геометрической прогрессии, получаем, что в (19) при $n \rightarrow \infty$

$$
p(s)=p^{[1 m]}(s)=\frac{b s}{1-(1-b) s}, \quad b=1-e^{\lambda_{2}} .
$$

Из (19) и (21) получаем, что

$$
\begin{gathered}
\frac{\delta \ln F(s)}{\delta s_{1}}=\frac{P}{k T} \frac{b}{[1-(1-b) s]^{2}}, \quad \frac{\delta^{2} \ln F(s)}{\delta s_{1} \delta s_{2}}=\delta\left(x_{1}-x_{2}\right) \frac{P}{k T} \frac{2 b(1-b)}{[1-(1-b) s]^{3}}, \\
\frac{\delta^{m} \ln F(s)}{\delta s_{1} \ldots \delta s_{m}}=\delta\left(x_{1}-x_{2}\right) \ldots \delta\left(x_{1}-x_{m}\right) \frac{P}{k T} \frac{m ! b(1-b)^{m-1}}{[1-(1-b) s]^{m+1}}, \quad m=3,4,5, \ldots .
\end{gathered}
$$

Полагая в первом из этих соотношений $s=1$, найдем, что $b=P / k T \rho$, где $\rho=$ $N / V$ - плотность числа частиц $N$. При $P / k T \rho>1$ надо сделать замену $b \rightarrow 1 / b$, результаты от этого не изменятся. Положив $s=0$, получим $z=(P / k T)^{2} / \rho$. Из второго соотношения получаем, что при $s=1$

$$
\rho_{12}^{(2)}-\rho_{1}^{(1)} \rho_{2}^{(1)}=\delta\left(x_{1}-x_{2}\right) \rho_{1}^{(1)} \rho_{2}^{(1)} \cdot 2\left(\frac{k T}{P}-\frac{1}{\rho}\right),
$$

где $\rho_{12}^{(2)}$ - двухчастичная функция распределения вида (15) при $m=2$ (вероятность того, что одна частица находится в окрестности точки $r_{1}$, а другая - в окрестности точки $\left.r_{2}\right) ; \rho_{i}^{(1)}, i=1,2$, - одночастичные функции распределения вида (15) при $m=1$, в однородном случае эти функции равны плотности $\rho$. При $s=0$ получаем

$$
b_{2} z^{2}=\frac{(P / k T)^{2}}{\rho}\left(1-\frac{P / k T}{\rho}\right) .
$$


Здесь и далее $b_{j}$ - групповые интегралы [33], [34]:

$$
\begin{aligned}
b_{2} & =\frac{1}{2 V} \iint f_{12} d r_{2} d r_{1} \\
b_{3} & =\frac{1}{6 V} \iiint\left(f_{31} f_{21}+f_{32} f_{31}+f_{32} f_{21}+f_{32} f_{31} f_{21}\right) d r_{1} d r_{2} d r_{3},
\end{aligned}
$$

где $f_{i j}=e^{-\beta \Phi_{2}\left(r_{i}-r_{j}\right)}-1$. Дальнейшие соотношения дают связи между групповыми интегралами. Такие же результаты можно получить для непрерывного случая и показательного распределения.

Для давления и плотности имеем конечные замкнутые выражения вида

$$
\begin{gathered}
\frac{P}{k T}=a=\frac{1-\left(1-4 \rho B_{2}\right)^{1 / 2}}{2 B_{2}}, \quad \rho=\frac{z}{\left(1+z B_{2}\right)^{2}}, \\
z=\frac{1-2 B_{2} \rho-\left(1-4 B_{2} \rho\right)^{1 / 2}}{2 \rho B_{2}^{2}}
\end{gathered}
$$

где $B_{2}=-b_{2}$ есть второй вириальный коэффициент [33], [34]. Первое соотношение в (23) представляет собой уравнение состояния в конечной замкнутой форме. В нем при $B_{2} \rightarrow 0$ для случая идеального невзаимодействующего газа, когда $\Phi_{2 i j}=0$, мы имеем $P / k T \rightarrow \rho$.

При конечных значениях параметра $n$ вместо (21) получим

$$
p(s)=p^{[1 m]}(s)=\frac{s\left(1-(q s)^{n}\right)(1-q)}{(1-q s)\left(1-q^{n}\right)}, \quad q=e^{\lambda_{2}}
$$

Из выражений для $\left.\frac{d p(s)}{d s}\right|_{s=0},\left.\frac{d^{2} p(s)}{d s^{2}}\right|_{s=0}$ и формулы (19) находим, что

$$
q=b_{2} z, \quad \frac{1-q}{1-q^{n}}=\frac{z}{a}, \quad q^{n}=1-\frac{a}{z}\left(1-b_{2} z\right), \quad n=\frac{\ln \left[1-(a / z)\left(1-b_{2} z\right)\right]}{\ln \left(b_{2} z\right)} .
$$

Из выражения для $\left.\frac{d p(s)}{d s}\right|_{s=1}$ находим, что плотность числа частиц равна

$$
\rho=\frac{a}{1-b_{2} z}\left[1-n\left(\frac{z}{a}-1+b_{2} z\right)\right]
$$

Так как

$$
\begin{gathered}
\iint\left(\rho_{12}^{(2)}-\rho_{1}^{(1)} \rho_{2}^{(1)}\right) d r_{1} d r_{2}=\left.V a \frac{d^{2} p(s)}{d s^{2}}\right|_{s=1}=\bar{N}\left[\frac{z}{\rho} \frac{d \rho}{d z}-1\right] \\
z \frac{d \rho}{d z}=\frac{\rho}{\bar{N}}\left(\left\langle N^{2}\right\rangle-\langle N\rangle^{2}\right),
\end{gathered}
$$


то из второй производной $\left.\frac{d^{2} p(s)}{d s^{2}}\right|_{s=1}$ от функции $(24)$ и производной $z \frac{d \rho}{d z}$ от плотности $\rho(25)$ находим еще одно уравнение, связывающее параметры $a, \rho, z, b_{2}$ :

$$
\left(\frac{\rho}{a}-1\right)\left[2-\frac{\rho}{a}\left(1-b_{2} z\right)-n \frac{z}{a}\right]+b_{2} z\left(n-\frac{\rho}{a}\right)+\left[z \frac{d n}{d z}-n(n-1)\right]\left[\frac{z}{a}-1+b_{2} z\right]=0,
$$

где

$$
z \frac{d n}{d z}=\frac{1}{\ln \left(b_{2} z\right)}\left[\frac{a b_{2}+b_{2}(\rho-a / z)-(\rho-a / z) / z}{1-a\left(1-b_{2} z\right) / z}-n\right] .
$$

Из соотношений (25) и (26) можно исключить параметр $z$ и записать уравнение состояния - связь $a=P / k T$ с $\rho$ через $b_{2}$. Из (25) и (26) получаем, что в отсутствии взаимодействий, когда $b_{2} \rightarrow 0$, мы имеем $n \rightarrow 1$, а $\rho \rightarrow a$, как и должно быть.

\section{3. УРАВНЕНИЕ СОСТОЯНИЯ ПРИ ИЗВЕСТНЫХ ПЕРВОМ И ВТОРОМ МОМЕНТАХ}

Если в дискретном случае известны нормировка, математическое ожидание $m$ и второй начальный момент, то полученное из функции Лагранжа распределение имеет вид

$$
p_{i}=\frac{e^{i\left(\lambda_{3} i+\lambda_{2}\right)}}{\sum_{k=1}^{n} e^{k\left(\lambda_{3} k+\lambda_{2}\right)}} .
$$

Производящая функция в (19) в этом случае равна

$$
p(s)=p^{[2 m]}(s)=\frac{\sum_{i=1}^{n} s^{i} e^{i\left(\lambda_{3} i+\lambda_{2}\right)}}{\sum_{k=1}^{n} e^{k\left(\lambda_{3} k+\lambda_{2}\right)}}=\frac{B(s)}{B},
$$

где

$$
B(s)=\sum_{k=1}^{n} s^{k} e^{k\left(\lambda_{3} k+\lambda_{2}\right)}, \quad B=\left.B(s)\right|_{s=1},
$$

a $\lambda_{2}, \lambda_{3}$ - множители Лагранжа из функционала Лагранжа, дополненного еще одним слагаемым,, соответствующие первому и второму моментам распределения (27).

ТЕОРЕма 1. Если производящая функиия $p(s)$ в выражении (19) записана в виде (28), а ПФ (7) и (19) совпадают, то связи между активностью $z$, плотностъю $\rho$ и давлением $a=P / k T$ имеют вид

$$
\begin{gathered}
z=\rho^{K /(K-1)} \frac{1}{m} \\
m \rho^{-1 /(K-1)} e^{-2 b_{2} a}=\left[\left(\frac{\rho}{a}-1\right) \frac{(a m)^{2}}{\rho^{K /(K-1)}\left(a m-\rho^{K /(K-1)}\right)}\right]^{b_{0}\left(1-\rho^{K /(K-1)} / m a\right)},
\end{gathered}
$$

где

$$
\begin{gathered}
m=\left[\left(a+\frac{\rho K}{b_{0}}\right) e^{-2 b_{2} a}\right]^{1 /(K-1)}, \quad K=b_{0} \frac{a}{a+b_{3} / b_{2}^{3}}, \quad a=\frac{P}{k T}, \\
b_{0}=\frac{1}{V^{2}} \iint_{V} y_{1 r} d r_{1} d r=1+\frac{2 b_{2}}{V}, \quad y_{1 r}=e^{-\Phi_{2}\left(r_{1}-r\right) / k T}=f\left(r_{1}-r\right)+1 .
\end{gathered}
$$


ДоКАЗАТЕЛЬСТВо. Подставляя (28) в (19) и используя (9) при $k=0,1,2,3$, находим, что

$$
\begin{gathered}
\lambda_{2}=\ln z+4 \ln b_{2}-\frac{3}{2} \ln b_{3}, \quad \lambda_{3}=\frac{1}{2} \ln b_{3}-\ln b_{2}, \\
B=a \frac{b_{2}^{3}}{b_{3}} .
\end{gathered}
$$

Выделив в числителе суммы (28) первое слагаемое, перепишем выражение (28) с учетом (8), (9), (30), (31) в виде

$$
p(s)=\frac{s e^{\lambda_{2}+\lambda_{3}}}{B}\left[1+B_{1} p_{1}(s)\right], \quad \frac{e^{\lambda_{2}+\lambda_{3}}}{B}=\frac{z}{a} .
$$

В данном случае в производящей функции $p_{1}(s)$ сделана замена $\lambda_{2} \rightarrow \lambda_{2}+2 \lambda_{3}$ и мы положили

$$
p_{1}(s)=\left.p(s)\right|_{\lambda_{2} \rightarrow \lambda_{2}+2 \lambda_{3}}, \quad B_{1}=\left.B\right|_{\lambda_{2} \rightarrow \lambda_{2}+2 \lambda_{3}} .
$$

Если в (32) заменить аргумент функции $s$ на $s_{1}=s e^{2 \lambda_{3}}$, то изменятся и производящие функции:

$$
p_{1}\left(s_{1}\right)=p(s) \frac{B}{B_{1}}, \quad p\left(s_{1}\right)=p_{2}(s) \frac{B_{2}}{B},
$$

где в производящей функции $p_{2}(s)$ проведена замена $\lambda_{2} \rightarrow \lambda_{2}-2 \lambda_{3}$ и мы положили

$$
p_{2}(s)=\left.p(s)\right|_{\lambda_{2} \rightarrow \lambda_{2}-2 \lambda_{3}}, \quad B_{2}=\left.B\right|_{\lambda_{2} \rightarrow \lambda_{2}-2 \lambda_{3}} .
$$

Соотношение (32) при замене $s$ на $s_{1}=s e^{2 \lambda_{3}}$ принимает вид

$$
\frac{B_{2}}{B} p_{2}(s)=s e^{-2 \lambda_{3}} \frac{z}{a}[1+B p(s)] .
$$

Из (30) получаем, что $e^{-2 \lambda_{3}}=b_{2}^{2} / b_{3}$. Полагая в $(32)$ и $(33) s=1$, получаем

$$
\begin{aligned}
& B_{1}=\frac{a}{z}-1, \\
& B_{2}=e^{-2 \lambda_{3}} B \frac{z}{a}(1+B) .
\end{aligned}
$$

В рамках предположения об однородности выражение (32) с учетом (19) переписывается в виде

$$
\ln F(s)=(s-1) a V+s\left(1-\frac{z}{a}\right) \ln F_{1}(s), \quad \ln F_{1}(s)=\int_{V} a\left(p_{1}(s)-1\right) d r .
$$

Аналогичные выражения записываются для (33) и других сходных, как (44), соотношений.

Используя формулы (7), (30), (19), (34), (35), запишем соотношения

$$
\begin{aligned}
& \frac{\partial \ln B}{\partial \lambda_{2}}=z \frac{d \ln B}{d z}=\left.\frac{\partial p(s)}{\partial s}\right|_{s=1}=\bar{k}=\frac{\rho}{a}, \\
& \frac{\partial \ln B_{1}}{\partial \lambda_{2}}=\left.\frac{\partial p_{1}(s)}{\partial s}\right|_{s=1}=\bar{k}_{1}=\frac{\rho_{(1)}}{a}=\frac{(a / z)(\rho / a-1)}{a / z-1}, \\
& \frac{\partial \ln B_{2}}{\partial \lambda_{2}}=\left.\frac{\partial p_{2}(s)}{\partial s}\right|_{s=1}=\bar{k}_{2}=\frac{\rho_{(2)}}{a}=1+\frac{B(\rho / a)}{1+B},
\end{aligned}
$$


где плотности $\rho_{(1)}, \rho_{(2)}$ соответствуют функции $(19)$ с $p_{1}(s), p_{2}(s)$ в правой части. Подставим в $(32) s=y_{1}$ и используем выражения (10), (11), (19). Тогда

$$
\begin{gathered}
\ln F\left(y_{1}\right)=\ln \frac{\rho}{z}=\int_{V} a\left(p\left(y_{1 r}\right)-1\right) d r, \quad \ln F_{1}\left(y_{1}\right)=\ln \frac{\rho_{(1)}}{z}, \\
p\left(y_{1}\right)=1+\frac{1}{a V} \ln \frac{\rho}{z} .
\end{gathered}
$$

В выражении для $p\left(y_{1}\right)$ предполагается однородность. Соотношение (32) с учетом (19) при $s=y_{1}$ после интегрирования по $d r_{1}$ и $d r$ и умножения на $V^{-1}$ принимает вид

$$
-2 b_{2} a+\ln \frac{\rho}{z}=b_{0}\left(1-\frac{z}{a}\right) \ln \left[\frac{a}{z} \frac{(a / z)(\rho / a-1)}{(a / z-1)}\right]
$$

где

$$
b_{0}=\frac{1}{V^{2}} \iint_{V} y_{1 r} d r_{1} d r=1+\frac{2 b_{2}}{V}
$$

Проделывая те же операции в равенстве (33), получаем еще одно выражение:

$$
-2 b_{2} a+\ln \left[\frac{a}{z}\left(1+\frac{B \rho / a}{1+B}\right)\right]=b_{0} \frac{B}{1+B} \ln \frac{\rho}{z} .
$$

С учетом (31) имеем

$$
\frac{B}{1+B}=\frac{a}{a+b_{3} / b_{2}^{3}} .
$$

Положим $K=b_{0} a /\left(a+b_{3} / b_{2}^{3}\right)$. Из (39) получаем

$$
z=\rho^{K /(K-1)}\left[\frac{e^{2 b_{2} a}}{a+\rho K / b_{0}}\right]^{1 /(K-1)} .
$$

Подставляя (40) в (38), получаем связь величин $a, \rho, b_{2}, b_{3}, b_{0}$, т. е. уравнение состояния

$$
\rho e^{-2 b_{2} a}=z\left[\left(\frac{a}{z}\right)^{2} \frac{\rho / a-1}{a / z-1}\right]^{b_{0}(1-z / a)}, \quad \text { где } \quad z=\rho^{K /(K-1)}\left[\frac{e^{2 b_{2} a}}{a+\rho K / b_{0}}\right]^{1 /(K-1)} .
$$

Когда взаимодействия нет, при $b_{2} \rightarrow 0, b_{0} \rightarrow 1, K \rightarrow 0$ (для случая идеального невзаимодействующего газа) из (40), (41) находим, что $\rho \rightarrow a=z$.

Положив $m=\left[\left(a+\rho K / b_{0}\right) e^{-2 b_{2} a}\right]^{1 /(K-1)}$, мы можем записать равенства $(40),(41)$ в виде (29). Теорема доказана.

Выделяя еще одно слагаемое в сумме (28), аналогично (32), (33) запишем соотношения

$$
\begin{gathered}
p(s)=s \frac{z}{a}\left[1+s z b_{2}\left(1+B_{3} p_{3}(s)\right)\right], \\
p_{4}(s) \frac{B_{4}}{B}=s \frac{z}{a} e^{-4 \lambda_{3}}\left[1+s e^{-4 \lambda_{3}} z b_{2}(1+B p(s))\right],
\end{gathered}
$$


где

$$
\begin{gathered}
B_{3}(s)=\left.B(s)\right|_{\lambda_{2} \rightarrow \lambda_{2}+4 \lambda_{3}}, \\
B_{3}=\left.B_{3}(s)\right|_{s=1}=\left.B\right|_{\lambda_{2} \rightarrow \lambda_{2}+4 \lambda_{3}}=\frac{B_{1}}{z b_{2}}-1=\frac{a-z\left(1+z b_{2}\right)}{z^{2} b_{2}}, \\
B_{4}(s)=\left.B(s)\right|_{\lambda_{2} \rightarrow \lambda_{2}-4 \lambda_{3}}, \\
B_{4}=\left.B_{4}(s)\right|_{s=1}=\left.B\right|_{\lambda_{2} \rightarrow \lambda_{2}-4 \lambda_{3}}=B e^{-4 \lambda_{3}} \frac{z}{a}\left(1+m_{1}\right), \\
m_{1}=e^{-4 \lambda_{3}} z b_{2}(1+B), \\
\rho_{3}=\frac{a(\rho+z-2 a)}{a-z\left(1+z b_{2}\right)}, \quad \rho_{4}=\frac{a}{1+m_{1}}\left(1+2 m_{1}+\frac{\rho K m_{1}}{a b_{0}}\right), \\
p_{k}(s)=\frac{B_{k}(s)}{B_{k}}, \quad k=1,2,3,4 .
\end{gathered}
$$

Полагая в (42), (43) $s=y_{1}$, получаем равенства, аналогичные (38), (39):

$$
\begin{gathered}
2 b_{2}\left[-a\left(1+b_{0}\right)+b_{0} z\right]+\ln \frac{\rho}{z}=b_{0}^{2}\left[1-\frac{z}{a}\left(1+z b_{2}\right)\right] \ln \left[\frac{a}{z} \frac{\rho-2 a+z}{a-z\left(1+z b_{2}\right)}\right], \\
-2 b_{2} a\left[1+\left(1+b_{0}\right) m_{1}\right]+\ln \left[\frac{a}{z} \frac{\left(1+2 m_{1}+\rho K m_{1} / b_{0} a\right)}{1+m_{1}}\right]=b_{0} m_{1} K \ln \frac{\rho}{z} .
\end{gathered}
$$

На основе этих соотношений и формул (38), (39) записываются моменты более высоких порядков (корреляционные функции) и алгебраические, в отличие от трансцендентных выражений (40), (41), уравнения, связывающие параметры $a, \rho, b_{2}, b_{3}, b_{0}$. Эти соотношения сложные, они представляют собой уравнения седьмого порядка. Основным результатом настоящей работы являются выражения (29), (40), (41).

ЗАмечАниЕ. Распределение (27) соответствует процессам Гаусса-Пуассона [13].

Можно записать непрерывное приближение, с нормальным распределением.

Согласно теореме Марцинкевича не существует случайных величин, характеристические показатели которых были бы полиномами выше второго порядка. Поэтому рассмотренные случаи исчерпывают все возможные ситуации.

\section{Список литературы}

[1] И. И. Иванчик, "Аналитическое представление уравнения состояния в классической статистической механике", ТМФ, 108:1 (1996), 135-158.

[2] E. T. Jaynes, "Information theory and statistical mechanics I.", Phys. Rev., 106:4 (1957), 620-630; "Information theory and statistical mechanics. II", Phys. Rev., 108:2 (1957), 171-190.

[3] P. Harremoës, F. Topsøe, "Maximum entropy fundamentals", Entropy, 3:3 (2001), 191-226; L. M. Martyushev, V.D. Seleznev, "Maximum entropy production principle in physics, chemistry and biology", Phys. Rep., 426:1 (2006), 1-45.

[4] Н. Н. Боголюбов, Проблемы динамической теории в статистической физике, Гостехиздат, М., 1946.

[5] Н.Н. Боголюбов (мл.), А.К. Прикарпатский, "Метод производящих функционалов Н.Н. Боголюбова в статистической механике и аналог преобразования к коллективным переменным", ТМФ, 66:3 (1986), 463-480. 
[6] Б. В. Мощинский, К. Родригес, В.К. Федянин, "Производящий функционал и функциональный аналог вариационного метода Боголюбова", ТМФ, 45:2 (1980), 251-260.

[7] Г. И. Назин, "Метод производящего функционала", Итоги науки и техн. Сер. Теор. вероятн. Матем. стат. Теор. кибернет., 22 (1984), 159-201.

[8] Г. И. Назин, "Описание гиббсовских случайных полей методом производящего функционала", ТМФ, 42:3 (1980), 383-391.

[9] В. В. Криволапова, Г. И. Назин, "Метод производящего функционала и гиббсовские случайные поля на счетных множествах", ТМФ, 47:3 (1981), 362-374.

[10] В. В. Рязанов, "Построение корреляционных функций сложной статистической системы", Изв. вузов СССР. Физика, 11 (1978), 129-130.

[11] В. В. Рязанов, "Модели производящего функционала статистической системы в области фазового перехода", Изв. вузов СССР. Физика, 9 (1983), 44-47.

[12] Б. А. Севастьянов, Ветвящиеся процессы, Наука, М., 1971.

[13] И.Н.Коваленко, Н. Ю. Кузнецов, В. М. Шуренков, Случайнъе прочессы. Справочник, Наукова думка, Киев, 1983.

[14] В. В. Рязанов, “Моделирование уравнения состояния пересыщенного пара и зависимости его равновесного давления от радиуса зародышевой капли", Журн. физ. химии, 58:1 (1984), 72-74.

[15] В. В. Рязанов, "Получение различных уравнений состояния вещества при помощи задания меры Леви обобщенного пуассоновского распределения", Теплофизика высоких mемператур, 21:6 (1983), 1099-1105.

[16] В. В. Рязанов, “Моделирование производящей функции для числа частиц ветвящимся процессом с иммиграцией", Физика жидкого состояния, Вып. 11, Вища школа, Киев, 1983, 40-44; "Моделирование термодинамических свойств гиббсовской статистической системы", Вып. 17, 1989, 28-41; "Конструктивное описание чистых веществ и смесей соотношениями типа уравнения Ван-дер-Ваальса", Вып. 18, 1990, 5-14; "Аналитическое моделирование систем Гиббса", Вып. 19, 1991, 24-35; В.В.Рязанов, О. К. Закусило, "Описание статистических систем при помощи случайного процесса гибели и иммиграции", Вып. 12, 1984, 85-93.

[17] Р. Л. Добрушин, "О законе Пуассона для распределения частиц в пространстве”, Украин. матем. журн., 8:2 (1956), 127-134.

[18] Р. Л. Добрушин, Ю. М. Сухов, "Временная асимптотика для некоторых вырожденных моделей эволюции систем с бесконечным числом частиц", Итоги науки и техн. Сер. Соврем. пробл. матем., 14, 1979, 147-254.

[19] Ю. М. Сухов, "Сходимость к пуассоновскому распределению для некоторых моделей движения частиц", Изв. АН СССР. Сер. матем., 46:1 (1982), 135-154.

[20] A. L. Rebenko, "Poisson measure representation and cluster expansion in classical mechanics", Commun. Math. Phys., 151:2 (1993), 427-435.

[21] D. J. Daley, D. Vere-Jones, An Introduction to the Theory of Point Processes, v. I: Elementary Theory and Methods, Springer, New York, Berlin, Heidelberg, 2002.

[22] Р. А. Минлос, “Лекции по статистической физике”, УМН, 23:1(139) (1968), 133-190.

[23] О. Л. Ребенко, В. А. Болух, "Нескінченновимірний аналіз і статистична механіка", Збірник праць Ін-ту математики НАН України, 11:1 (2014), 281-339.

[24] D. L. Finkelshtein, Y. G. Kondratiev, M. J. Oliveira, "Glauber dynamics in the continuum via generating functionals evolution", Complex Anal. Oper. Theory, 6:4 (2011), 923-945.

[25] V. A. Boluh, O. L. Rebenko, "An exponential representation for some integrals with respect to Lebesgue-Poisson measure", Methods Funct. Anal. Topol., 20:2 (2014), 186-192.

[26] Д. Фінкельштейн, “Стохастичні динаміки неперервних систем”, Міждисциплінарні дослідження складних систем, 6 (2015), 5-48. 
[27] О.Е. Ланфорд, “Эволюция во времени больших классических систем”, Гиббсовские состояния в статистической физике, Математика. Новое в зарубежной науке, 11, ред. Р. А. Минлос, Мир, М., 1978, 159-218; англ. пер.: О. Е. Lanford III, "Time evolution of large classical systems", Dynamical Systems, Theory and Applications, Lecture Notes in Physics, 38, ed. J. Moser, Springer, Berlin, Heidelberg, 1975, 1-111.

[28] D. Ruelle, "Superstable interactions in classical statistical mechanics", Commun. Math. Phys., 18:2 (1970), 127-159.

[29] A. L. Rebenko, "Cell gas model of classical statistical systems", Rew. Math. Phys., 25:4 (2013), 1330006, 28 pp.

[30] A. L. Rebenko, M. V. Tertychnyi, "On stability, superstability and strong superstability of classical systems of statistical mechanics", Meth. Funct. Anal. Topology, 14:3 (2008), $287-296$.

[31] К. Престон, Гиббсовские состояния на счетных множествах, Мир, М., 1977.

[32] H.-O. Georgii, Canonical Gibbs measure, Lecture Notes in Mathematical Physics, 760, Springer, Berlin, 1979; Х.-О. Георги, Гиббсовские меры и фазовые переходь, М., Мир, 1992.

[33] Т. Хилл, Статистическая механика, ИЛ, М., 1960.

[34] Дж. Майер, М. Гепперт-Майер, Статистическая механика, Мир, М., 1980.

[35] S. Albeverio, Yu. G. Kondratiev, M. Röckner, "Analysis and geometry on configuration spaces: the Gibbsian case", J. Funct. Anal., 157:1 (1998), 242-291.

[36] Y. G. Kondratiev, J. L. Silva, L. Streit, "Differential geometry on compound Poisson space", Methods Funct. Anal. Topology, 4:1 (1998), 32-58, arXiv: math/9908059.

[37] А. М. Вершик, И. М. Гельфанд, М. И. Граев, "Представления группы диффеоморфизмов", УМН, 30:6(186) (1975), 3-50.

[38] В. И. Тихонов, М. А. Миронов, Марковские процессъ, Советское радио, М., 1977.

Поступила в редакцию 10.01.2017, после доработки 22.03.2017 